\title{
ON THE ASSOCIATION OF THE LIFELENGTHS OF COMPONENTS SUBJECTED TO A STOCHASTIC ENVIRONMENT
}

\author{
CLAUDE LEFEVRE, * Université Libre de Bruxelles \\ XAVIER MILHAUD, ${ }^{* *}$ Université des Sciences et Techniques du Languedoc
}

\begin{abstract}
This note is concerned with a system of non-renewable components in parallel subjected to a common environment which is described by a real-valued external stochastic process. Given the environment process, the components are supposed to work independently, and the corresponding failure rates are all increasing (or decreasing) functions of the observed current state. It is then proved that, under these assumptions, the association of the external process implies the association of the component lifelengths. Connection with existing results is underlined.
\end{abstract}

FAILURE RATE; ASSOCIATED VARIABLES

\section{Introduction}

Consider a system of $N$ non-renewable components in parallel, and let $T_{i}$ denote the lifelength of component $i, i=1, \cdots, N$. For reasons of simplicity, it is often assumed that the different components of the system operate independently. In reality, however, there are a number of situations with some form of dependence between the lifelengths. A usual factor inducing correlation is the random environment that affects all the components of the system. Lindley and Singpurwalla (1986) and Lefèvre and Malice (1989) investigated a model where the environment acts at the initial instant only and the joint lifelengths are then distributed as a mixture of $N$ independent exponential laws. Here, an alternative approach is followed by representing the environment as a real-valued stochastic process $Y=\left\{Y_{t}, t \geqq 0\right\}$ which is external (or exogeneous) to the failure mechanism.

Specifically, we assume the following. The environment process $Y$ has real-valued cadlag trajectories. Given $Y$, the lifelengths $T_{i}$ are independent and

$$
\lim _{\tau \rightarrow 0}(1 / \tau) P\left[t<T_{i} \leqq t+\tau \mid T_{i}>t, Y\right]=\xi_{i}\left(t, Y_{t}\right), \quad i=1, \cdots, N,
$$

where each $\xi_{i}(t, y)$ is a positive continuous function of $t \in \mathbb{R}_{+}$and $y \in \mathbb{R}$. Further, for the purposes of this paper, we shall assume that $\xi_{i}(t, y)$ are all increasing (or decreasing) in $y$. Observe that given the external process $Y$, the failure rates depend on $Y$ only through the current state $Y_{i}$. However, $Y$ could be redefined to include cumulative measures describing the history of changes in the environment at the cost of complicating the state space of $Y$. We point out that in a similar context, Çinlar and Özekıci (1987) introduced a more tractable model of this type where the failure rates depend on the whole history of $Y$ via the so-called intrinsic age process of each component. In its present formulation, the model above extends

Received 10 May 1990; revision received 25 July 1990.

* Postal address: Institut de Statistique, CP 210, Université Libre de Bruxelles, Boulevard du Triomphe, B-1050, Bruxelles, Belgique.

The paper was written while the author was visiting the Institut de Mathématiques de l'Université des Sciences et Techniques du Languedoc.

** Postal address: Institut de Mathématiques, Université des Sciences et Techniques du Languedoc, Place Eugène Bataillon, F-34060 Montpellier, France. 
the one examined by Lefèvre and Michaletzky (1990) in the special case of a birth-and-death environment.

It is clear that the components' lifelengths now become correlated, and our goal is to characterize the nature of that dependence. Under the hypotheses above, we prove that the association of the environment process $Y$ implies the association of the lifelengths $T_{i}$ (as well as the association of the risks incurred by each component). Recall that the (real) random variables $T_{1}, \cdots, T_{N}$ are associated (in the sense of Esary et al. (1967)) if

$$
\operatorname{cov}\left[f\left(T_{1}, \cdots, T_{N}\right), g\left(T_{1}, \cdots, T_{N}\right)\right] \geqq 0
$$

for all increasing functions $f$ and $g$ for which the covariance exists; the (real-valued) stochastic process $Y$ is said to be associated if the variables $Y_{t_{1}}, \cdots, Y_{t_{k}}$ are associated for all choices of the integer $k \geqq 1$ and times $t_{1}, \cdots, t_{k}$.

That association property of the $T_{i}$ reinforces and extends Theorem 2 of Lefèvre and Michaletzky (1990). A similar result has also been derived, inter alia, by Çinlar et al. (1989) for the model of Çinlar and Özekici (1987). The proof given here is elementary and may be of some value since it puts in evidence that the result can be viewed as a natural extension of a theorem of Jogdeo (1978) on the association of mixtures of associated random variables.

\section{Association of the lifelengths}

We begin by noting that, in view of the hypothesis (1), each conditional survival function $P\left(T_{i}>t \mid Y\right)$ satisfies the ordinary differential equation

$$
d P\left(T_{i}>t \mid Y\right)=-P\left(T_{i}>t \mid Y\right) \xi_{i}\left(t, Y_{t}\right) d t, \quad i=1, \cdots, N .
$$

Assuming that the lifetimes are positive with probability one, (3) can be solved 'path by path' and gives

$$
P\left(T_{i}>t \mid Y\right)=\exp \left[-\int_{0}^{t} \xi_{i}\left(u, Y_{u}\right) d u\right], \quad i=1, \cdots, N .
$$

Note that, by hypothesis, $Y$ has cadlag paths and the $\xi_{i}(t, y)$ are continuous in $y$, which together imply that $\xi_{i}\left[u, Y_{u}(\omega)\right]$ is a cadlag function of $u$ for each possible outcome $\omega$ in the underlying probability space. Hence, the right-hand side of (4) is well defined as the exponential of a Lebesgue integral.

Next, we put as Lemma 1 a result due to Jogdeo (1978) that gives conditions under which mixtures of associated random variables remain associated. Recall that a vector $V$ is said to be stochastically increasing in the (real) vector $W$ if $E[f(V) \mid W=w]$ is increasing in $w$ for all positive increasing functions $f$.

Lemma 1. Let $W$ be an associated random vector, and $V$ be a random vector that is conditionally associated given $W$ and stochastically increasing (or decreasing) in $W$. Then, $V$ is associated.

The following result is useful for what follows and has been proved by Ahmed et al. (1981), amongst others.

Lemma 2 . Let $V_{1}, \cdots, V_{N}$ be random variables that are conditionally independent (hence associated) given $W$ and suppose that each is stochastically increasing in $W$. Then $V=\left(V_{1}, \cdots, V_{N}\right)$ is stochastically increasing in $W$.

Comparing the actual model with the situation considered by Jogdeo (1978), we see that the role of the vector $W$ is played here by the external process $Y$ and the vector $V$ corresponds to the lifelengths $\left(T_{1}, \cdots, T_{N}\right)$ (subjected to the environment $Y$ ). The proposition below gives sufficient conditions for the (unconditional) association of the $T_{i}$. As expected, these conditions can be viewed as a natural extension of those obtained by Jogdeo (1978). 
Proposition 3. Under the assumptions of the model specified earlier, if the environment process $Y$ is associated, then the lifelengths $T_{1}, \cdots, T_{N}$ are associated.

Proof. Let $t \geqq 0$ be any fixed time and $T$ be the random vector $\left(T_{1} \wedge t, \cdots, T_{N} \wedge t\right)$. By the dominated convergence theorem, the association of the $T_{i}$ is equivalent to the association of the $T_{i} \wedge t$; that is, $\operatorname{cov}[f(T), g(T)] \geqq 0$ for all increasing functions $f$ and $g$. Moreover, without loss of generality, we can take in the covariance $f$ and $g$ increasing, bounded and continuous (see Esary et al. (1967)). Now, consider the time interval $[0, t]$ and, for simplicity in the notations, put $Y$ as the restriction of the external process $Y$ on $[0, t]$. Conditionally on $Y$, the $T_{i} \wedge t$ are assumed to be independent, hence associated, so that

$$
\begin{aligned}
E[f(T) g(T)] & \geqq\{E[f(T) \mid Y] E[g(T) \mid Y]\} \\
& \equiv E[b(Y) c(Y)], \text { say. }
\end{aligned}
$$

Thus, for our purpose, it suffices to prove the association of $b(Y)$ and $c(Y)$. From the assumptions of the model, $b(Y)$ can be written as

$$
b(Y)=\int_{[0, t]^{N}} f\left(u_{1}, \cdots, u_{N}\right) \prod_{i=1}^{N} F_{i}\left(d u_{i} \mid Y\right),
$$

where $F_{i}(u \mid Y), u \in \mathbb{R}_{+}$, represents the conditional distribution function of $T_{i} \wedge t$ given $Y$ and is directly obtained from (4). A similar expression holds true for $c(Y)$. Following then a standard procedure, let us approximate $Y$ by the sequence of processes $Z_{n}=\left\{Z_{n, u}, 0 \leqq u \leqq t\right\}$, $n \geqq 1$, where

$$
Z_{n, u}=\sum_{k=0}^{2^{n}-1} Y_{k t / 2^{n}} 1_{\left[k t / 2^{n},(k+1) t / 2^{n}\right)}(u) \text {. }
$$

$Z_{n}, n \geqq 1$, is a cadlag process, and as $n \rightarrow \infty, Z_{n}$ converges a.s. to $Y$ in $D[0, t]$ for the Skorohod topology. Observe from (4) and (6) that $b$ and $c$ are continuous functionals on $D[0, t]$. This implies that $b\left(Z_{n}\right)$ and $c\left(Z_{n}\right)$ converge a.s. to $b(Y)$ and $c(Y)$ as $n \rightarrow \infty$, respectively. Therefore, our problem reduces to that of showing the association of $b\left(Z_{n}\right)$ and $c\left(Z_{n}\right)$ for all $n \geqq 1$. Let $y$. and $z_{n}$. be trajectories of $Y$ and $Z_{n}$ respectively. From (7) and since the process $Y$ is assumed to be associated, we have just to prove that $b\left(z_{n .}\right)$ and $c\left(z_{n}\right)$ are both increasing (or decreasing) in the $y_{k t / 2^{n}}$. Coming back to the definition of these two functions and then applying Lemma 2 with $V_{i}=T_{i} \wedge t, i=1, \cdots, N$, we deduce that the latter property will be satisfied if each variable $T_{i} \wedge t$ is stochastically increasing (or decreasing) in the $Y_{k t / 2^{n}}$. From (4), this is clearly true when the functions $\xi_{i}(t, y)$ are all decreasing (or increasing) in $y$, which completes the proof.

We note that the condition for $Y$ to be associated is verified, for instance, for (real-valued) stochastically monotone Markov processes (see, e.g., Barlow and Proschan (1975)). A simple particular case is the birth-and-death environment process considered by Lefèvre and Michaletzky (1990).

For each component $i$, let us now introduce the random variable

$$
Q_{i}\left(t_{i}\right)=\int_{0}^{t_{1}} \xi_{i}\left(u, Y_{u}\right) d u, \quad t_{i} \geqq 0, \quad i=1,, \cdots, N .
$$

From (4), it is legitimate to interpret $Q_{i}\left(t_{i}\right)$ as the total risk incurred by component $i$ from the initial instant until time $t_{i}$. The dependence between these different risks is examined below.

Proposition 4. Under the assumptions of Proposition 3, the risks $Q_{1}\left(t_{1}\right), \cdots, Q_{N}\left(t_{N}\right)$ are associated.

Proof. First, observe that $Q_{i}\left(t_{i}\right), i=1, \cdots, N$, can be expressed as the a.s. limit, as $n \rightarrow \infty$, 
of the Riemann sums

$$
S_{i, n}=\frac{1}{2^{n}} \sum_{k=0}^{\left[t_{i} 2^{n}\right]-1} \xi_{i}\left(k / 2^{n}, Y_{k / 2^{n}}\right), \quad n \geqq 1 .
$$

It suffices thus to prove the association of the $S_{i, n}$ for all $n \geqq 1$. This follows directly from (9) and the hypotheses made.

The result is, of course, intuitive. Incidentally, a straightforward consequence is that the $T_{i}$ are positively orthant dependent; this property, however, has been reinforced by Proposition 3.

\section{Acknowledgment}

We wish to thank the referee for many valuable comments that led to improvements in the paper.

\section{References}

AhMed, A.-H. N., LeÓN, R. AND Proschan, F. (1981) Generalized association, with applications in multivariate statistics. Ann. Statist. 9, 168-176.

Barlow, R. E. AND Proschan, F. (1975) Statistical Theory of Reliability and Life Testing: Probability Models. Holt, Rinehart and Winston, New York.

ÇinLaR, E. AND Özexici, S. (1987) Reliability of complex devices in random environments. Prob. Engrg. Inform. Sci. 1, 97-115.

Çinlar, E., Shaked, M. and Shanthikumar, J. G. (1989) On lifetimes influenced by a common environment. Stoch. Proc. Appl. 33, 347-359.

Esary, J. D., Proschan, F. AND Walkup, D. W. (1967) Association of random variables, with applications. Ann. Math. Statist. 38, 1466-1474.

JoGDEO, K. (1978) On a probability bound of Marshall and Olkin. Ann. Statist. 6, 232-234.

LEFEVRE, C. AND MALICE, M.-P. (1989) On a system of components with joint lifetimes distributed as a mixture of independent exponential laws. J. Appl. Prob. 26, 202-208.

LEFEVRE, C. AND MichaleTZKY, G. (1990) Interparticle dependence in a linear death process subjected to a random environment. J. Appl. Prob. 27, 491-498.

Lindley, D. V. AND SingPuRwalla, N. D. (1986) Multivariate distributions for the life lengths of components of a system sharing a common environment. J. Appl. Prob. 23, 418-431. 